\title{
Percolative conductivity and critical exponents in mixed-valent manganites
}

\author{
Ye Xiong, ${ }^{1}$ Shun-Qing Shen, ${ }^{2,3}$ and X. C. Xie ${ }^{1}$ \\ ${ }^{1}$ Department of Physics, Oklahoma State University, Stillwater, Oklahoma 74078 \\ ${ }^{2}$ Department of Physics, The University of Hong Kong, Pokfulam, Hong Kong, China \\ ${ }^{3}$ Institute of Physics, Chinese Academy of Sciences, P.O. Box 603, Beijing 100080, China
}

(Received 8 December 2000; published 23 March 2001)

\begin{abstract}
Recent experiments have shown that some colossal magnetoresistance (CMR) materials exhibit a percolation transition. The conductivity exponent varies substantially with or without an external magnetic field. This finding prompted us to carry out theoretical studies of percolation transition in CMR systems. We find that the percolation transition coincides with the magnetic transition, and therefore the magnetic field has a large effect on the percolation transition. Using a real-space-renormalization method and numerical calculations for twodimensional (2D) and three-dimensional (3D) models, we obtain the conductivity exponent $t$ to be 5.3 (3D) and 3.3 (2D) without a magnetic field, and 1.7 (3D) and 1.4 (2D) with a magnetic field.
\end{abstract}

DOI: 10.1103/PhysRevB.63.140418

PACS number(s): 75.90.+w, 71.27.+a

Colossal magnetoresistance (CMR), an unusual large change of resistivity in the presence of a magnetic field, has been extensively studied in ferromagnetic perovskite manganites. ${ }^{1}$ It is well known that the electronic phase diagrams of CMR materials are very complex. There are various ordering states and phase transitions as the carrier concentration is varied. For example, $\mathrm{La}_{1-x} \mathrm{Ca}_{x} \mathrm{MnO}_{3}$, a typical double exchange ferromagnetic metal when $x=0.33$, becomes a charge ordered insulator with a specific type of electronic orbital and magnetic orderings when $x=1 / 2 .^{2}$ Recently, it was demonstrated that a $\mathrm{La}_{1-x-y} \mathrm{Pr}_{y} \mathrm{Ca}_{x} \mathrm{MnO}_{3}(x=3 / 8)$ system, where Pr is chosen to vary the chemical pressure, may be electronically phase separated into a submicrometer-scale mixture of insulating regions and ferromagnetic (FM) metallic regions. ${ }^{3,4}$ Experimental findings of FM clusters and phase separation in CMR materials have also been reported in early studies. ${ }^{5}$ Electron diffraction and dark-field imaging on the $\mathrm{La}_{1-x-y} \mathrm{Pr}_{y} \mathrm{Ca}_{x} \mathrm{MnO}_{3}$ samples indicate that the insulating region is a $x=1 / 2$ charge ordered phase. This is not a charge congregation type of phase separation, which was observed in slightly doped antiferromagnetic manganites ${ }^{6}$ and was extensively discussed. ${ }^{7}$ The CMR effect was observed in different samples with $0.275<y<0.41$, and was explained by percolative transport through the ferromagnetic domains. According to the percolation theory, the conductivity $\sigma \propto(p$ $\left.-p_{0}\right)^{t}$, where $p$ is the concentration of the metallic phase and $p_{0}$ is its critical value. In Ref. 3 , the exponent was studied in the presence and absence of an external magnetic field and two values are substantially different. Thus, the experiment shows that the percolative transport in the CMR systems depends sensitively on the relative spin orientation of adjacent ferromagnetic domains which is controlled by an applied magnetic field. The goal of this work is to develop a percolation theory which takes into account the magnetic transition in the CMR materials. In particular, we would like to understand what causes the exponent to be so different with or without an external magnetic field.

Based on phenomenological considerations, we study two- or three-dimensional (2D or 3D) lattice percolation models. The conductivity and its critical exponent are calculated by means of real-space renormalization and numerical methods. The system consists of three types of lattice sites. Each site has spin $S_{i}(= \pm 1,0)$ where $i$ denotes the index of a site. This model is similar to the site-diluted spin system used in Ref. 8. $S_{i}=0$ implies that the site is empty, meaning occupied by the $x=1 / 2$ charge ordered phase. $S_{i}= \pm 1$ means the site is occupied by the ferromagnetic metallic phase with up and down magnetizations. ${ }^{9}$ The Hamiltonian of the spin interaction is Ising-like and is written as

$$
H_{s}=-J \sum_{\langle i, j\rangle} S_{i} \cdot S_{j}+\sum_{i} H \cdot S_{i}
$$

Here $\langle i, j\rangle$ denotes a pair of the nearest-neighbor sites, $J$ is the interaction energy, and $H$ is the strength of the external magnetic field. We consider a ferromagnetic interaction, i.e., $J>0$. In order to investigate transport properties, we assume that the local conductivity between two nearest occupied sites is either 0 for antiparallel spins $(\uparrow \downarrow$ and $\downarrow \uparrow)$ or 1 for parallel spins ( $\uparrow$ and $\downarrow \downarrow)$ and is zero if one or both sites are empty. Thus, the conductivity between neighboring sites can be expressed as follows

$$
\sigma_{i j}= \begin{cases}1, & S_{i} \cdot S_{j}=1 ; \\ 0, & \text { otherwise }\end{cases}
$$

First, we use the standard Monte-Carlo method to study the magnetic properties of the model. Knowing the spin structure is necessary for the transport studies since the local conductivity depends on the spins through Eq. (2). The conductance calculation is performed on a set of spin configurations produced by the Markov chain. In the Markov chain every spin configuration is generated from the previous one by using the probability $e^{-\beta \Delta H_{s}} /\left(e^{-\beta \Delta H_{s}}+1\right)$, where $\Delta H_{s}$ is the energy difference between these two configurations and $\beta=1 / k_{B} T .{ }^{11}$ The calculations are carried out on finite square and cubic lattices for $2 \mathrm{D}$ and $3 \mathrm{D}$ systems and the periodic boundary condition is adopted to eliminate the boundary effects. The conductance $G$ for every spin configuration in the Markov chain is obtained by calculating the total conductance of the resistor network. ${ }^{10}$ In the resistor network, the local conductivity between neighboring sites is determined 

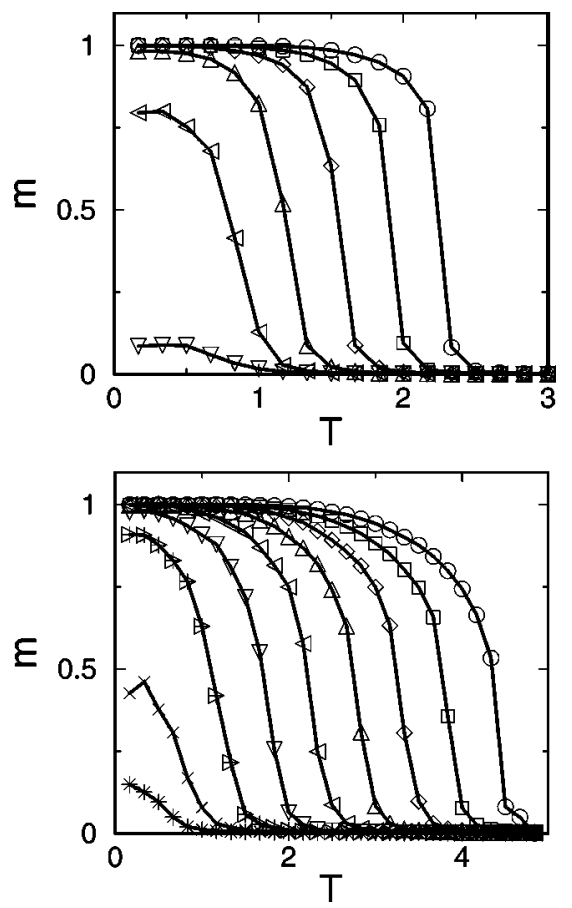

FIG. 1. (a) The normalized spontaneous magnetization $m$ as a function of temperature $T$ for different concentration $p$ in the 2D model. The unit of $T$ is set by the interaction strength $J / k_{B}$. From top to bottom, the concentrations are $p=1.0,0.9,0.8,0.7,0.6$, and 0.5 The calculation is performed on 1000 samples whose sizes are $100 \times 100$. (b) $m$ as a function of $T$ in the 3D model. The calculation is performed on 200 samples whose sizes are $10 \times 10 \times 10$. From top to bottom, the concentrations are $p=1.0,0.9,0.8,0.7,0.6,0.5$, $0.4,0.3$, and 0.2 .

by Eq. (2). The total conductance is calculated for many samples to obtain the average conductance.

When the charge order (CO) phase is dominant in some of the CMR materials, most sites are empty according to our model. Thus, most of the conductivities between neighboring sites are zero, corresponding to the low concentration limit $(p \sim 0)$ in the percolation model, where $p$ is the probability of nonzero local conductivity. The small FM islands are well separated by the $\mathrm{CO}$ phase. Because the spin correlations between FM blocks are cut off by the CO phase, the spin orientation for each FM block is random, either up or down. Therefore, the spontaneous magnetization $m$ will be zero at any temperature $T$. As $p$ increases, $m$ continues to be zero until $p$ reaches $p_{0}$ at which the first infinite FM cluster appears. If $p>p_{0}$, a finite spontaneous magnetization appears for $T<T_{c}(p)$ with $T_{c}(p)$ the critical temperature at concentration $p$. In Figs. 1(a) and 1(b) we plot the normalized magnetization $m$ as a function of temperature $T$ with different concentration probabilities $p$ for 2D and 3D systems, respectively, calculated using the Monte-Carlo method. The interaction strength $J$ is set to unity. It can be seen that when the temperature increases $m$ is reduced and reaches zero at $T_{c}(p)$. From magnetization data with different size samples and through finite-size scaling, we can determine the critical temperature. In Figs. 2(a) and 2(b) we plot the relation between the critical temperature $T_{c}(p)$ and the concentration $p$.
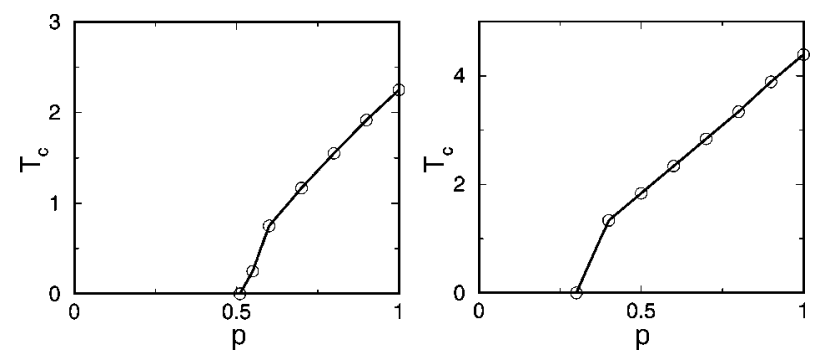

FIG. 2. (a) The critical temperature $T_{c}$ as a function of the concentration $p$ in 2D. (b) The critical temperature $T_{c}$ as a function of the concentration $p$ in $3 \mathrm{D}$.

As shown in these plots, $T_{c}(p)$ will approach the Curie temperature $T_{c}$ for the regular Ising model as $p$ approaches 1 . We find that $T_{c}$ determined from Fig. 2 is $2.3 \mathrm{~J}$ or $4.4 \mathrm{~J}$ for a 2D or 3D system. Both of them are in good agreement with the values of the regular Ising model. ${ }^{12}$

From the spin configurations of the system, we can calculate the conductance by using the local conductivity defined in Eq. (2). In a paramagnetic phase, there is no infinite cluster with spins pointing in the same direction. Hence, according to Eq. (2) there is no conducting path throughout the sample. On the other hand, the first conducting path appears simultaneously when the magnetization starts becoming nonzero. Thus, the phase transition from the FM phase to the paramagnetic phase is accompanied by the metal-insulator (MI) transition in the conductance. This implies that at zero temperature the MI transition occurs at the critical concentration of the percolation threshold, $p_{0}$. Near the critical point the averaged conductance $G$ can be expressed as $G$ $\sim\left(p-p_{0}\right)^{t}$, where $t$ is the conductance critical exponent for the transition.

We now discuss the real-space renormalization method. We start with a 2D system and later extend to a more complicated 3D system. The essential physics does not depend on dimensionality, although numerical numbers do. Consider a triangular lattice in a $2 \mathrm{D}$ plane. The choice of a triangular lattice is for the convenience of rescaling of the system in the real-space renormalization procedure. By enlarging the system by factor $\sqrt{3}$ and grouping every three sites into a "supersite," the number of the supersites is the same as the number of sites in the original system (see Fig. 3). In Fig. 3, the thin lines are for the original system and the thick lines are for the rescaled system. A supersite is regarded as empty if the majority of the three sites is empty. Thus the concentration of the system can be expressed as $p^{\prime}=p^{3}+3 p^{2}(1$ $-p$ ) (Ref. 13). Near the critical point $p_{0}=0.5$, on the metallic side, $G$ can be written as $G=G_{0}\left(p-p_{0}\right)^{t}$, where the constant $G_{0}$ is proportional to the conductance of the unit cell. For the enlarged system this relation becomes $G^{\prime}=G_{0}^{\prime}\left(p^{\prime}\right.$ $\left.-p_{0}\right)^{t}$. In a $2 \mathrm{D}$ system the conductance is independent of the system size and we have $G=G^{\prime}$. Thus, $t=\ln \left(G_{0} / G_{0}^{\prime}\right) / \ln \left[\left(p^{\prime}\right.\right.$ $\left.\left.-p_{0} / p-p_{0}\right)\right]$. If the spin degree of freedom is frozen, the conductance of elementary cell is inversely proportional to the size of the cell, hence, $t=\ln (\sqrt{3}) / \ln \left(\frac{3}{2}\right)=1.35$. This value is very close to the exact value of $t=4 / 3$ in the standard 2D percolation model. ${ }^{14}$ When taking into account the spin de- 


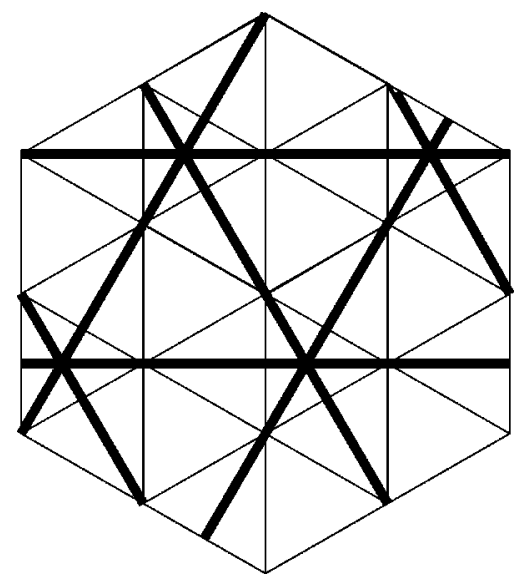

FIG. 3. Illustration of renormalization in a $2 d$ triangle lattice.

grees of freedom, as we discussed above, the $p_{0}$ point is also the critical point for the spontaneous magnetization. The conductance $G_{0}$ (or $G_{0}^{\prime}$ ) is now associated with spin configurations. The spin dependence comes from the fact that $G_{0}$ is proportional to the average of conductivity between two nearest-neighbor sites which is spin dependent,

$$
\begin{aligned}
& G_{0} \sim\left\langle\sigma_{i j}\right\rangle \sim\left\langle\cos \left(\frac{\theta_{i}-\theta_{j}}{2}\right)\right\rangle \\
&=\frac{1}{A} \int_{0}^{\pi} d \theta_{i} \int_{0}^{2 \pi} d \phi_{i} \int_{0}^{\pi} d \theta_{j} \int_{0}^{2 \pi} d \phi_{j} \\
& \times e^{-\beta m S \cos \theta_{i}} e^{-\beta m S \cos \theta_{j}} \cos \left(\frac{\theta_{i j}}{2}\right) .
\end{aligned}
$$

The conductivity expression between the nearest-neighbor spin $\sigma_{i j} \sim \cos \left(\theta_{i j} / 2\right)$ comes from the double exchange model where $\theta_{i j}$ is the angular difference between spins $S_{i}$ and $S_{j}$, which satisfies ${ }^{15}$

$$
\cos \theta_{i j}=\cos \theta_{i} \cos \theta_{j}+\sin \theta_{i} \sin \theta_{j} \cos \left(\phi_{i}-\phi_{j}\right) .
$$

$\left(\theta_{i}, \phi_{i}\right)$ denotes the orientation of $\operatorname{spin} S_{i} . A$ is a normalization constant. From the above equation, it is easy to show that $G_{0} \sim m^{2}$. Because $m$ can be written as $m \sim\left(p-p_{0}\right),{ }^{13}$ we finally get the conductance critical exponent in $2 \mathrm{D}$ triangular lattice as $t^{\prime} \simeq 1.35+2=3.35$.

In the $3 \mathrm{D}$ case, we consider the normal cubic lattice. The elementary vectors of the enlarged lattice is just two times of those of the original one $\left\{\hat{e}_{x}{ }^{\prime}, \hat{e}_{y}{ }^{\prime}, \hat{e}_{z}{ }^{\prime}\right\}=\left\{2 \hat{e}_{x}, 2 \hat{e}_{y}, 2 \hat{e}_{z}\right\}$. In this case $p^{\prime}=\sum_{n=4}^{8} C_{8}^{n} p^{n}(1-p)^{8-n}$, from which we conclude that $p_{0}=0.395$ and $t=1.7$ without spin effects. After the spin degrees of freedom are considered, the formula $G_{0}$ $\sim m^{2}$ is still satisfied. But in a $3 \mathrm{D}$ case $m$ has $m \sim(p$ $\left.-p_{0}\right)^{1.79} .^{13}$ So the critical exponent $t^{\prime} \simeq 1.7+2 \times 1.79 \simeq 5.3$.

In Fig. 4 we show the numerical results of $2 \mathrm{D}$ conductance $G$. The 3D calculation has not been done because of computational limit. The points with the steepest drop are defined as the critical points for the metal-insulator transition. These points are consistent with the magnetic critical points (see Fig. 1), as we discussed before. In the inset of

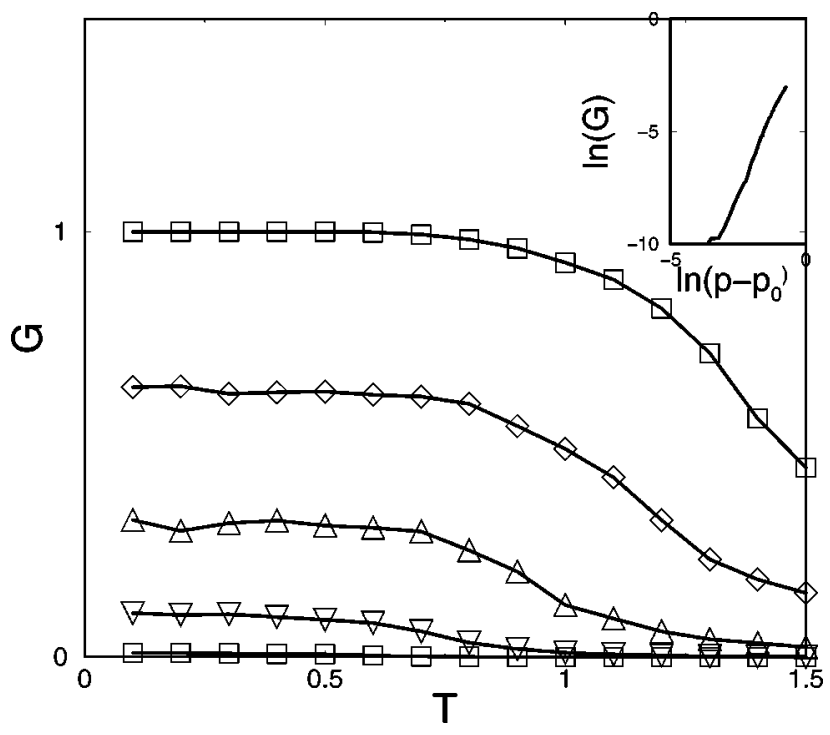

FIG. 4. The normalized conductivity $G$ as a function of temperature $T$ in a $2 \mathrm{D}$ model. From top to bottom, the concentrations are $p=1.0,0.9,0.7,0.6$, and 0.5. Inset: $\log G$ vs $\log \left(p-p_{0}\right)$ shows a linear behavior with a slope $\simeq 3.5$.

Fig. 4 we show the $\ln (G)$ versus $\ln \left(p-p_{0}\right)$. They exhibit a linear dependence and the slope of the curve, corresponding to the exponent $t$, is roughly 3.5. This value is in good agreement with the estimation from the renormalization group consideration although it is obtained from a different type of lattice structure. In Table I we list the results of this work and compare them with the previous studies of the standard percolation theory ${ }^{14}$ and experimental exponents in CMR materials. ${ }^{3,4}$ The experimental exponent $t_{3}$ (or $t_{3}^{\prime}$ ) was obtained in the absence (or presence) of an external magnetic field.

Before summary, we would like to make a couple of comments. (i) In order to calculate the conductivity exponent, we have to determine the dependence of $G_{0}$ [see Eq. (3)] on the magnetization $m$, and the double exchange model is used to achieve that goal. However, the double exchange may not be the origin of the ferromagnetism in doped manganites, as shown in a recent work. ${ }^{16}$ This might explain some of the discrepancy between the experimental and our theoretical values in the conductivity exponent. We should also mention that the values from the two experimental groups are not in

TABLE I. The critical exponent $t$ for $2 d$ and $3 d$ cases. $p_{c 2}$ and $p_{c 3}$ are percolation threshold for $2 d$ and $3 d$, respectively. $t_{d}$ and $t_{d}^{\prime}$ are the critical exponents for the $d$-dimension model without or with spin effects. Previous results are from Ref. 14. Experiment I is from Ref. 3 and Experiment II is from Ref. 4.

\begin{tabular}{lcccccc}
\hline \hline & $p_{c 2}$ & $p_{c 3}$ & $t_{2}$ & $t_{3}$ & $t_{2}^{\prime}$ & $t_{3}^{\prime}$ \\
\hline Previous results & 0.5 & 0.31 & 1.33 & 1.9 & & \\
Experiment I & & & & 2.6 & & 6.1 \\
Experiment II & & & & & & 2.6 \\
Renormalization method & 0.5 & 0.37 & 1.35 & 1.7 & 3.3 & 5.3 \\
Numerical results & 0.51 & 0.32 & 1.30 & & 3.5 & \\
\hline \hline
\end{tabular}


good agreement with each other as seen in Table I. (ii) We have neglected the quantum effects in this work. This might be justified because $T_{c}$ is relatively high in these samples. Developing a semiclassical transport theory in this problem is a difficult task because of the finite-phase coherence length. There are attempts ${ }^{17}$ of using the semiclassical theory to understand the two-dimensional metal-insulator transition.

In summary, we argue that the percolation threshold corresponds not only to the appearance of an infinite metal cluster, but also to the phase transition from PM to FM. This coincidence of two phase transitions renormalizes the critical exponents. The conductivity exponent has been obtained using real-space renormalization and numerical calculations. The exponent is found to be quite different whether the magnetic transition is considered. This finding explains the large exponent discrepancy in some of the CMR materials in the presence or absence of an external magnetic field.

This work was supported by DOE and a RGC grant of Hong Kong. We thank Junren Shi for many helpful discussions.
${ }^{1}$ See, Colossal magnetoresistive Oxides, edited by Y. Tokura (Gordon and Breach Sciences Publishers, New York, 2000).

${ }^{2}$ P. Schiffer, A. P. Ramirez, W. Bao, and S. W. Cheong, Phys. Rev. Lett. 75, 3336 (1995).

${ }^{3}$ M. Uehara, S. Mori, C. H. Chen, and S. W. Cheong, Nature (London) 399, 560 (1999).

${ }^{4}$ N. A. Babushkina et al., Phys. Rev. B 62, R6081 (2000).

${ }^{5}$ G. M. Zhao, H. Keller, J. Hofer, A. Shengelaya, and K. A. Müller, Solid State Commun. 104, 57 (1997); M. R. Ibarra et al., Phys. Rev. B 57, 7446 (1998).

${ }^{6}$ G. Allodi et al., Phys. Rev. B 56, 6036 (1997); Hennion et al., Phys. Rev. Lett. 81, 1957 (1998).

${ }^{7}$ A. Moreo, S. Yunoki, and E. Dagotto, Science 283, 2034 (1999), and references therein.

${ }^{8}$ E. Marinari et al., Phys. Rev. B 62, 4999 (2000).

${ }^{9}$ To simplify our problem, we just take two possible orientations for magnetization of ferromagnetic domains in the mixtures.

${ }^{10}$ B. Derrida and J. Vannimenus, J. Phys. A 15, L557 (1982).

${ }^{11}$ M. Suzuki, Quantum Monte Carlo Methods in Condensed Matter Physics (World Scientific Publishing, Singapore, 1993).

${ }^{12}$ See, for example, M. Plischke and B. Bergersen, Equilibrium Statistical Physics (World Scientific, Singapore, 1989).

${ }^{13}$ A. Bunde and S. Havlin, Fractal and Disordered Systems (Springer-Verlag, Berlin, 1996).

${ }^{14}$ B. I. Shklovskii and A. L. Efros, Electronic Properties of Doped Semiconductors (Springer-Verlag, Berlin, 1984).

${ }^{15}$ See, for example, E. L. Nagaev, Physics of Magnetic Semiconductors (Moscow, Mir Publishing, 1979).

${ }^{16}$ G. M. Zhao, Phys. Rev. B 62, 11639 (2000).

${ }^{17}$ J. R. Shi and X. C. Xie, Phys. Rev. B 63, 045123 (2001); J. R. Shi, S. He, and X. C. Xie, cond-mat/9904393 (unpublished). 